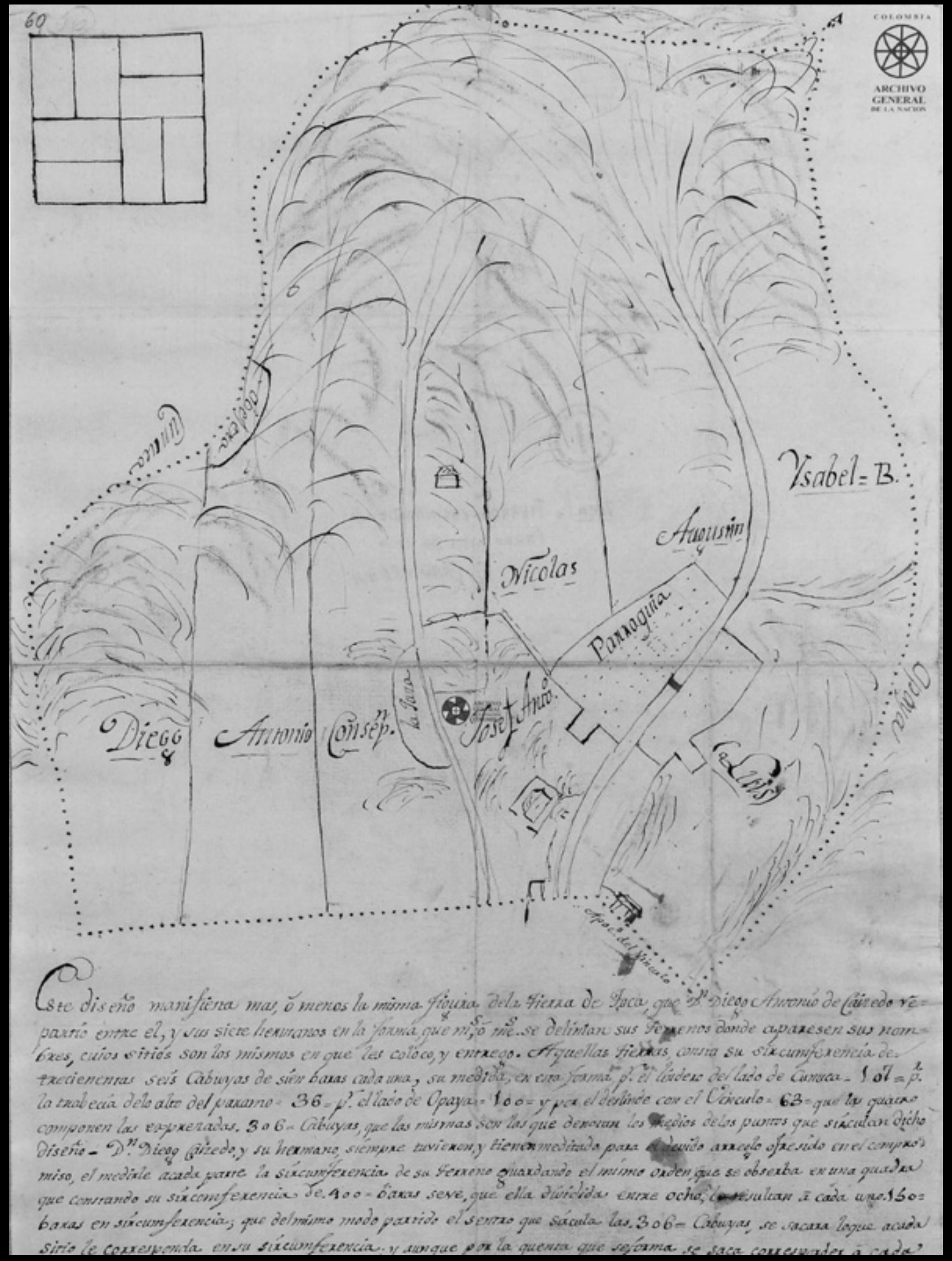




\section{Espacialidad de la provincia de Tunja en el siglo xIx: \\ representaciones políticas y geográficas}

Spatiality of the Tunja Province in the 19TH Century: Political and Geographical Representations

Espacialidade da província de Tunja no século XIX: representações políticas e geográficas

\section{Daniel Roberto Vega Torres}

Instituto de Investigaciones científicas en

Educación INICIEN Educación

(Tunja, Colombia)

Doctorando en en Ciencias Sociales Aplicadas de la Universidade Estadual de Ponta Grossa, Brasil.

\section{Resumen}

Este artículo contextualiza de manera espacial a la provincia de Tunja a mediados del siglo xix. Se busca presentar mediante la configuración política y geográfica un proceso de construcción histórica de la provincia. Se analiza un espacio concebido de dominio territorial mediante la cartografía regional, un espacio percibido desde los discursos académicos de la geografía universal, y, por último, un espacio vivido desde experiencias socioculturales. Estos tipos de representación de la espacialidad ofrecen un grado de comprensión particular de Tunja dentro del contexto neogranadino.

\section{Palabras clave}

espacialidad; provincia de Tunja; historia regional

\section{Abstract}

This article compraises a spacial contextualization of the Tunja Province in the MID-NINETEENTH century. It seeks to present, through its political and geographical setting, a historical construction process of the province. It analyzes a space designed for territorial control through regional mapping, a space perceived from the academic discourses of the universal geography, and, finally, a space lived from socio-cultural experiences. These types of spatiality representation offer a particular understanding of Tunja within the neogranadinian context.

\section{Keywords}

spatiality; Tunja province; regional history
La investigación deriva del proyecto "Sociedad de Instrucción Popular: sociabilidad política liberal de Tunja 1838-1854", inscrito en el Grupo de investigación en Pedagogía y Humanidades COL0100903, con apoyo institucional de la Fundación Universitaria Juan de Castellanos.

\section{Resumo}

Este artigo contextualiza de maneira espacial a província de Tunja em meados do século XIX. Mediante a configuração política e geográfica, visa-se apresentar um processo de construção histórica da província. Analisa-se um espaço concebido de domínio territorial mediante cartografia regional, um espaço percebido desde os discursos académicos da geografia universal, e, por fim, um espaço vivido desde experiências socioculturais. Estes tipos de representação da espacialidade oferecem um grau de compreensão particular de Tunja dentro do contexto neogranadino.

\section{Palavras-chave}

espacialidade; província de Tunja; história regional 


\section{Introducción}

Este documento presenta el análisis espacial de la provincia de Tunja como una forma de identificación de representaciones históricas diferente a las «naturalizaciones» político-administrativas ${ }^{1}$. Propende por estudiar representaciones que se examinen más allá de la concepción de un espacio dado con un orden institucionalizado, mediante una reflexión que permita entender el espacio como producto y producción social. Para el caso, se propone el reconocimiento de Tunja a mediados del siglo xIX como una construcción histórica que implica diversidad de representaciones políticas y geográficas de consolidación del territorio provincial. Este periodo de frecuentes conflictos y cambiantes organizaciones políticas adquiere un valor significativo para comprender cómo un espacio social se genera de distintas visiones y territorialidades que se transponen en un tiempo determinado.

El espacio social puede concebirse como representación o producto social, histórico y cultural, definido desde las contingencias, posibilidades y necesidades de la vida en sociedad. Con el fin de desarrollar esta reflexión se parte de la categoría espacialidad. Para Edward Soja ${ }^{2}$ la espacialidad, entendida como categoría «trialéctica» de un espacio concebido, percibido y vivido, acoge la relación vital y puede entenderse como una condición existencial de los seres sociales. Esta relación deviene como una práctica social que permite analizar un espacio concebido, es decir, identificar y diseñar la producción del espacio de manera que impone un «orden» al conocimiento, el cual se codifica, ejerciendo una fuerza en las relaciones sociales. De la misma manera, como una representación subjetiva o cognitiva, un espacio percibido que asegura la continuidad y la cohesión mediante actos que permiten la reiteración y construcción de la misma práctica social puede presentarse como la construcción imaginada. Por último, se reflexiona sobre un espacio vivido,

1 Clara Cortés de Sarmiento, Dimensión espacial de Boyacá: geografía (Tunja: Academia Boyacense de Historia, 2008). Instituto Geográfico Agustín Codazzi, Características geográficas Boyacá (Bogotá: IGAC, 1990).

2 Edward W. Soja, Thirdspace: Journeys to Los Angeles and other Real-and-Imagined Places (Oxford: Blackwell, 1996). lugar «real-imaginado», una forma clandestina de la vida social, fuera de la percepción rutinaria y que se acerca más a la expresión artística.

Así, en este artículo se contextualiza históricamente la espacialidad de la provincia de Tunja a mediados del siglo XIX tanto en las representaciones políticas y gubernamentales en el interior, como la forma en que se clasificaba e imaginaba a la provincia dentro del estudio geográfico universal. En la primera parte se presenta la manera como se construye la visión territorial de la provincia con el fin de obtener un dominio del territorio como espacio concebido bajo el gobierno de Francisco José de Hoyos en 1843. En segundo lugar, se presenta la comprensión de un espacio percibido por habitantes y extranjeros en donde se rescata como objetivo de estudio y de dominio tanto de forma cultural en el desarrollo de investigaciones del pueblo muisca, como de las intenciones de explotación de minas de esmeraldas en muzo. Por último, se presenta una expresión del espacio «real-imaginado» como forma narrativa de las costumbres y experiencias de individuos en la provincia. Estos tipos de representación de la espacialidad ofrecen un grado de comprensión que contribuye a entender por qué la situación de Tunja llegaba a presentar aspectos contingentes en el desarrollo del contexto neogranadino y cómo puede estudiarse conjuntamente.

\section{Cartografía de la provincia como espacio concebido}

La cartografía en la actual región boyacense ha tenido grandes aportes durante el siglo $\mathrm{XIX}^{3}$, con proyecciones de diverso grado y materia, lo que hace un foco de interés sus interpretaciones y formas de comprensión para la historiografía. Sin embargo, para este caso iniciaré este apartado desde una comprensión más localizada para luego exponer la organización de la provincia a mediados del siglo xix. Se concibe el espacio desde un tipo de «racionalidad» más definible y delimitada a partir del caso de la parroquia de Toca, en la Provincia de Tunja, a inicios de dicho siglo. La

3 Martha Martínez y Luz Marina Bautista, Cartografía histórica de los territorios boyacenses (Tunja: Banco de la Republica, 2003). 
imagen que sirve de portadilla para este artículo presenta un mapa de la parroquia de Toca ${ }^{4}$-entendiéndose la denominación «parroquia» como una forma político-administrativa de los territorios que se constituían por «blancos» $\mathrm{y}$ «libres de todos los colores», a diferencia de los habitantes de los pueblos ${ }^{5}$. Este documento expresa la división del terreno que Diego Antonio de Caicedo hace junto con sus siete hermanos. En contraste con otros mapas y láminas de la época, esta división más que «describir» el territorio para su administración expresa fundamentalmente su dominio y posesión.

La importancia del documento, a parte de su destacada intención de mostrar el paso del latifundio al minifundio y la división de tierras fuera del mayorazgo, es la manera como se calcula el espacio, como se divide, de tal manera que pueda concebirse un valor de relativa igualdad y proporcionalidad para la distribución de este por transferencia de privilegios dentro de una familia. El total del área de diseño es de 306 cabuyas $^{6}$, delimitada por los puntos con el fin, como se lee en el mapa, de «medirle a cada parte la sircunferencia de su terreno guardando el mismo orden que se observa en una quadra que constando su sircunferencia de 400 baras se ve que ella dividida entre ocho, le resultan a cada uno 150 baras en sircunferencia; que al mismo modo partido el sentro que sircula las 306 cabuyas se sacara lo que a cada sitio le corresponda [sic]».

La imagen de un cuadrado dividido en ocho rectángulos proporcionales que aparece en la parte superior expresa la búsqueda de una medición deseable, de una distribución que cosifica la tierra como un objeto diseñado, concebido, imaginario,

4 Archivo General de la Nación (AGN), Tierras vecinas a la Parroquia de Toca (1806), Mapoteca, Smp. 4, Ref. 482 A.

5 Marta Herrera Ángel, «Las divisiones político-administrativas del virreinato de la Nueva Granada a finales del período colonial», Historia Crítica (2001).

6 Para comprender la dimensión y medidas de la cabuya, se puede anotar un documento de 1761 en la provincia de Girón: «[...] la cabuya medida, según va referido, compone cien pasos, de forma que en siendo el señalamiento de un pueblo tres mil pasos, se le miden treinta cabuyas; y por lo que mira al término desde donde se comienza a medir, en unos pueblos se medía desde la última casa de lo poblado y en otros desde la puerta de la iglesia, dando cinco cabuyas o seis para el asiento del pueblo». Víctor Manuel Patiño, La tierra en la América Equinoccial, Libro iv (Bogotá: Presidencia de la República, 1997). que puede tomarse, usarse, dividirse. No quiere decirse con ello que sea una «nueva» racionalidad en la época, ni tampoco que no haya existido antes este tipo de representaciones, sino que con este mapa se ejemplifica cómo desde la provincia de Tunja se producen otras formas de representación política del territorio. Es decir, desde contextos locales se contienen mecanismos de diseño del territorio que pueden equiparase a intereses no solo familiares, sino a llegar a constituir límites y fronteras para el uso, acceso y dominio de un territorio específico por regiones. Así, entendida desde estos intereses de concepción y diseño del espacio, el objetivo de comprender la configuración de la provincia de Tunja durante el siglo xix puede advertirse desde la manera como se consolidan procesos de gobierno regionales relativamente autónomos y que permiten un esfuerzo de imaginar lo que para ellos era «su» posesión. Durante los fuertes cambios de organización política que se generaron en las primeras décadas del siglo, el proceso de consolidación regional se ve afectado por disputas caudillistas internas y procesos de consolidación del poder derivados de los representantes provinciales de la élite tunjana en el gobierno central. Aunque esta sea solo una parte de la división territorial, diferente de la que pudo darse en los pueblos de indios y en algunas parroquias y villas, en general se consolida la región como una propuesta tanto interna como externa para el control administrativo y de hacienda.

Luego de los disturbios presentados a inicios de la década de los cuarenta en la llamada guerra de los supremos, la posesión de Francisco J. de Hoyos le ofreció a la provincia de Tunja un cierto grado de estabilidad política, pues su discurso venía a ser conciliador y propositivo. Paralelo a la reorganización de un Estado bajo el centralismo, impulsado por la propuesta política de Márquez, Herrán y Mosquera, la provincia de Tunja tomó un camino de reorganización de la política pública luego de las batallas libradas en su suelo. El gobierno de Hoyos se había proclamado partidario del orden «ministerial», había apoyado la derrota de los reaccionarios y se sentía augusto con el apoyo del clero. La disposición de una nueva carta constitucional dejó claros los derroteros por los cuales iba a transitar el gobierno durante la década, pues la expulsión y control de 
los rebeldes caudillos regionales había provocado una derrota clara de las aspiraciones de los bandos «exaltados» del liberalismo.

La población de la provincia de Tunja, según censo de 1843, se encontraba distribuida de la siguiente manera: Cantón de Tunja 34.752, de Soata 28.589, del Cocuy 25.836, de Garagoa 16.028, de Santa Rosa 34.647, de Leiva 20.011, de Tenza 31.415, de Ramiriqui 36.942 y de Sogamoso $43.553^{7}$. Para esa fecha, la división judicial de la Nueva Granada estaba dispuesta por 7 distritos, los cuales se responsabilizaban de algunas provincias. La provincia de Tunja, junto con la de Vélez y Casanare, estaba circunscrita por el distrito judicial de Boyacá que se ubicaba en la ciudad de Tunja con 3 magistrados a su cargo. De los distritos judiciales se derivan los circuitos judiciales a cargo de jueces encargados por Cantón.

Para 1843, la gobernación presentaba el informe anual a la cámara de provincia, en el que se hacía referencia a las acciones desarrolladas durante el último año, iniciando con el orden social del cual menciona que su propuesta de reconciliación había permitido domeñar las revueltas y que la provincia se encontraba en un estado de calma. Para ese entonces, de los 271.703 habitantes de la provincia solo treinta eran esclavos, sin embargo se proyectaba su manumisión con fondos del gobierno. El estado de los hospitales era aún precario, en especial el de la ciudad, Hospital de caridad San Juan de Dios de Tunja, pero mantenía sus servicios activos, proporcionando a lo largo de los últimos años cama, alimentos, medicinas y auxilios.

En relación con los fondos derivados de las rentas provinciales, la carencia era notable, lo que visualizaba los escasos recursos con que contaba la provincia para poder mejorar las condiciones de la población. La proyección de nuevos impuestos, especialmente de licores, por su impacto moral era una de las propuestas del gobernador para la cámara con el fin de mejorar el haber de la gobernación. El colegio de Boyacá seguía con actividades normales, se reportaba la presencia en el último año escolar de 53 cursantes o estudiantes de gramática latina, idiomas vivos, filosofía,

7 Estadística Jeneral de la Nueva Granada. Parte Primera (Bogotá: BNC, Fondo Pineda 486, 1846). agricultura y mineralogía. Sumado al Colegio de Boyacá se establece en 1842 la Escuela Normal Superior, con proyección a toda la provincia.

El estado de las cárceles era deplorable, en especial por la falta de mantenimiento y por su espacio, «Estrechísima, i por lo mismo mui mal sana, serviría únicamente para enfermar a los que son reducidos a ella, si el estado de ruina en que se encuentra no les facilitase el escarpar desde que se proponen a hacerlo» ${ }^{8}$. De igual manera las vías de comunicación eran escasas para el momento y se proyectaba que con apoyo de los jefes de cantón se llevará a cabo en los años siguientes la conexión con provincias cercanas como las de Vélez, Socorro y Casanare, al igual que fortalecer las vías de comunicación internas entre los cantones.

Pese a la precaria situación de la provincia en ámbitos como los hospitales, el comercio y la renta, la novedad en la administración fue la presentación de la «carta corográfica de la provincia», hecho que sobresale en la administración de Hoyos y presenta un interés peculiar por lograr identificar el territorio, de definir una forma de visualizar e imaginar lo que es la provincia de Tunja, pese a la variabilidad y dinámica política. La carta corográfica fue una propuesta del gobernador desde el año anterior (1842) y fue envidada al presidente Herrán el 29 de marzo de 1843. Su objetivo era claro: la identificación de las tierras de la provincia como una forma de concretar los avances constitucionales del año anterior, permitiendo estabilizar el país y la provincia. Así es como Hoyos justificó su propuesta:

La verdad sea dicha, una nueva división territorial de la República no podrá arreglarse con acierto sin que el lejislador tenga a la vista el mapa jeneral de la Nueva Granada, i las cartas particulares de cada una de sus provincias: es absolutamente preciso que su estención del territorio sea esplorada, reconocida y examinada para que el lejislador pueda combinar, pesar i modificar en la balanza política las ventajas de cada comarca, i con relación a su centro; ventajas que nunca podrán valuarse sino se conoce el país jeográficamente9.

Este motivo o visión de gobierno es lo que identifica las expectativas de la consolidación del poder

\footnotetext{
8 Memoria del gobernador de Tunja a la cámara provincial (Bogotá: BNc, Fondo Pineda 733, pza. 25, 1843), 11. 9 Memoria del gobernador de Tunja, 15-16.
} 
pretendiendo un tipo de gobierno mucho más «ilustrado» al reconocer las posibilidades y capacidades si se sabe dividir o controlar el terreno. Este tipo de estrategia ilustrada se veía iniciada desde finales del siglo xvin, gracias a la reorganización política de los territorios, en especial de pueblos y parroquias ${ }^{10}$. Es reconocible que los mapas no solo tenían un fin político, «este tipo de documentos llegaba a un reducido círculo compuesto por los sectores letrados del país pero, de otra parte, en numerosos casos, los textos estaban destinados a la enseñanza en escuelas primarias de la Nueva Granada»"

La carta corográfica, a diferencia de otras imágenes que se hayan producido de la provincia, tiene el valor de sistematizar aspectos de medición y cálculo que anteriormente no se tenían y que, por el contrario, para una provincia era una novedad, sobre todo luego de la crisis social en que hacía un par de años se encontraba la administración estatal y provincial. En cierta medida la propuesta cartográfica, como espacio concebido o diseñado, condensaba las ideas gubernamentales, que si bien se pretendía por el orden ministerial, no puede negar su aporte a la actividad de gobierno de la provincia permitiendo llevar a cabo mecanismos de accesibilidad a los pueblos y cantones, asegurando que el diseño promoviera la comunicación con otras provincias de la Nueva Grana$\mathrm{da}$, consolidando mercados y rutas.

Junto a la forma de representación centrada en el cálculo topográfico, en el naturalismo aséptico a las desviaciones políticas y sociales, a ese objetivo ilustrado que pervivió en la formación de la Gran Colombia, se distancia la representación regional de la Nueva Granada, donde cantones y provincias intensifican su importancia mediante el límite, la puesta en marcha de la identidad territorial. Volvemos a la definición de lo regional y lo nacional como un diálogo complementario para la racionalidad de la administración pública y de los recursos territoriales que ella dispone.

10 Francisco Antonio Moreno y Escandón, Indios y mestizos de la Nueva Granada a finales del siglo xviII Transc. Germán Colmenares y Alonso Valencia (Bogotá: Biblioteca Banco popular, 1985), 133.

11 Lucía Duque Muñoz, «El discurso geográfico y cartográfico colombiano sobre los límites entre Nueva Granada y Venezuela (18301883)», Anuario Colombiano de Historia Social y de la Cultura 36, n. 1 (2009): 133.

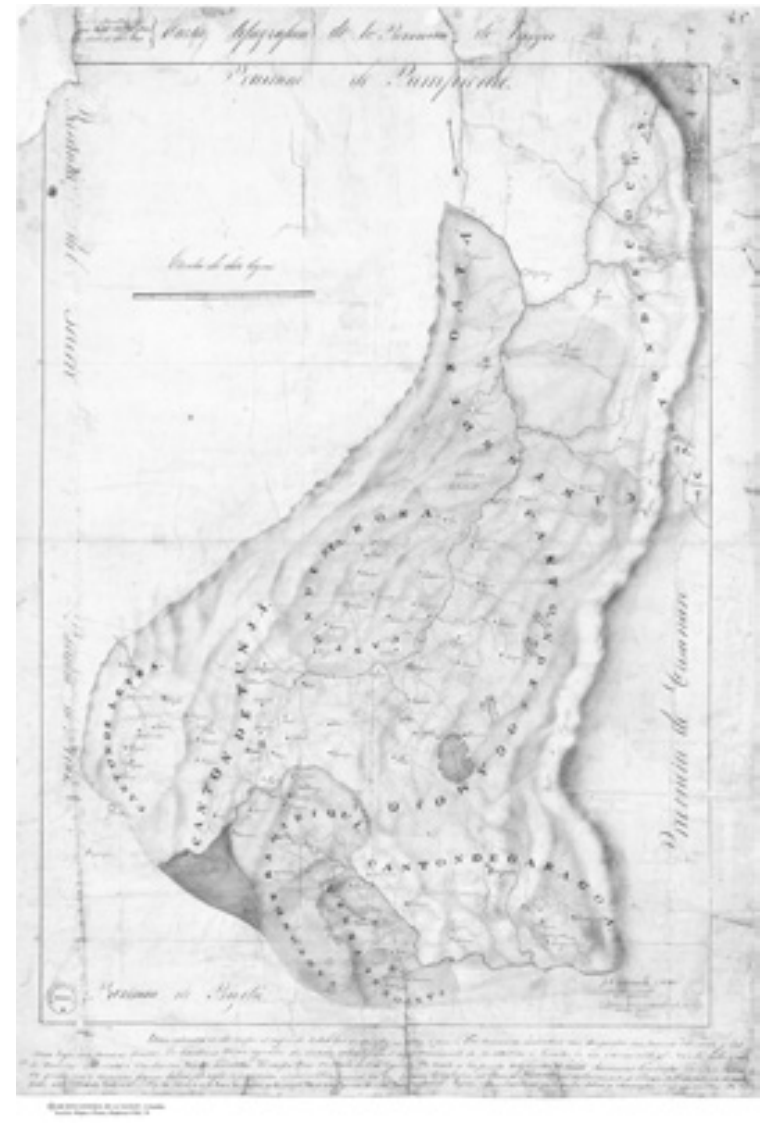

Figura 1. Carta geográfica de la provincia de Tunja (1842-1843)

Fuente: J. F. M. a expensas de Francisco José de Hoyos, lo hizo marzo 15 de 1843. En colores 89 X $63 \mathrm{~cm}$. Escala de 10 leguas. Archivo General de la Nación (AGN), Mapoteca 6, ref. 91. Núm. 1814²

Así, como menciona Carlos Rincón y Volker Lühr, «La parcelación administrativa idealmente homogénea que propone la administración territorial moderna, en la cual todos los habitantes y todas las partes del territorio serían iguales, suprime el origen de usurpación violenta del territorio sin dejar por eso de basarse en él» ${ }^{13}$.

12 Se puede leer en el mapa: «Están colocados en este mapa el origen de todas las vertientes su curso i su fin, las serranías más atrás van designadas con sombras oscuras y las más bajas en sombras más densas. Los cantones llevan aguadas de diversos colores para el mayor conocimiento de su estensión i límites, los ríos i demás vertientes van de color azul, los caminos provisionales i veredas con puntos amarillos. El mapa lleva su escala de diez leguas, su norte y los puntos adyacentes de cuatro provincias limítrofes. Las tres líneas de grados que le cupieron fueron hechas por regla de proporción aproximativa tomando por par la carta topográfica del Varón de Humboldt, del departamento de Boyacá. Cada cabecera de cantón tiene una estatuilla colorada. Hay seis salinas, la de la Galera en el cantón de Ramiriquí, tres en el cantón de Sogamoso llamadas Zisbaque, Sismosá i Sirguasa, i dos en el Cocui que se llaman, Salina y Chinivaque. El lugar de la Salina Tiene una cruz».

13 Carlos Rincón y Volker Lühr, «Del poder y la geografía: La cartografía como fuente de legitimación en Colombia» (Disertación inaugu- 
Esa naturalización de la vida política representada dentro de la imagen de los cantones es la pertinencia del gobierno para dividir el territorio de tal forma que se produzca un sentido de proporcionalidad y justicia, y ante todo cristalización de la posesión de la tierra por parte del notablato. Tendría que esperarse que los intereses de la representación cartográfica se centralizaran para poder obtener una idea de complementariedad de las regiones del país, pues las propuestas de representación provincial y cantonal dispuestas desde el mandato de Francisco de Paula Santander no serían lo suficientemente estables y continuas para asociarlas a otras regiones y poder ser integradas en un todo estatal.

Este tipo de representación política del territorio emerge de manera interna para responder a la necesidad de dominio de los cantones y pueblos, fortaleciéndose de manera clara en un primer momento con los aportes separados de cada gobernación durante la primera mitad del siglo ${ }^{14}$, pero que con la consolidación de la comisión corográfica abarcan de manera más amplia la configuración del Estado republicano, dirigiéndose a un tipo de representación técnicamente más consolidada ${ }^{15}$. No obstante, las representaciones socioespaciales de la provincia de Tunja y de la Nueva Granada pueden integrarse a procesos de medición y clasificación provenientes desde el exterior y que permiten construir una mirada conjunta de los intereses de otros sujetos en la definición del territorio y cómo puede la provincia de Tunja proyectarse más allá de las vicisitudes políticas y conflictos caudillistas, frecuentes en el siglo xIX.

\section{Geografía universal como espacios percibidos}

Por otro lado, para la década de los cuarenta, los procesos de comunicación de la situación política

ral para la obtención del título de doctor en la Facultad de Filosofía y Humanidades, Universidad Libre de Berlín, 2006), 65-66.

14 Lucía Duque Muñoz, «Geografía y cartografía en la Nueva Granada Producción, clasificación temática e intereses», Anuario Colombiano de Historia Social y de la Cultura 33 (2006): 18.

15 Archivo General de la Nación (AGN), Provincia de Tunja. Mapa corográfico de la provincia de Tunja, levantado por orden del gobierno por Agustín Codazzi, 1850. 64×68. Escala: 1: 340.000, Mapoteca: smp 6, Ref. 20. mundial atravesaban por formas de representación académica que permitían la visualización de las condiciones de vida en otros lugares del mundo. Sin embargo, cuestionar la manera como desde afuera se observaba a la República de la Nueva Granada, y qué aspectos constituían el imaginario europeo y norteamericano frente a las condiciones sociales, económicas y geográficas de zonas como Tunja es algo que pasa desapercibido en la historiografía nacional. En este apartado se observa no solo esas visiones de extranjeros en la Nueva Granada ${ }^{16}$, sino que además se visualiza la idea de «Nueva Granada» $y$ «Tunja» desde la literatura científico-académica de Francia y Norteamérica a mediados del siglo xix, esto con el fin de reconocer a la provincia de Tunja como un espacio percibido; igualmente, se reflexiona sobre la alteridad de la investigación histórica del territorio, teniendo en cuenta que la mirada del extranjero tiene unos códigos y símbolos que clasifican, miden y proyectan frente a su propia representación del mundo, por ejemplo desde aquello que es civilizado y aquello que no ${ }^{17}$.

La idea de llevar a cabo un proceso de formalización de la disciplina geográfica principalmente con aspectos políticos fue uno de los planteamientos de la actividad académica francesa durante el siglo xix. El proceso de organización de la interpretación política y física del mundo tuvo un horizonte en la geografía luego de los procesos de formación republicana. Es desde allí donde puede entreverse la necesidad de observar lo universal como categoría de lo humano en la corriente ilustrada de inicios del siglo xIx. Ya sea por la idea del gobierno de la representación visual como estrategia política, el resultado de la puesta en marcha de un programa de Geografía universal permitiría un proceso de visualización y estudio de otros lugares del mundo.

Una de las primeras empresas académicas con este sentido es la obra de Conrad Malte-Brun o Malthe Conrad Bruun (1775-1826) de 1803 a 1807, Géographie mathématique, physique et politique de toutes

16 Puede consultarse para este fin: Jaime Jaramillo Uribe, «La visión de los otros. Colombia vista por observadores extranjeros en el siglo XIX», Historia crítica 24 (2003).

17 Ingrid Bolívar Ramírez, «Los viajeros del siglo xIx y el "proceso de la civilización": Imágenes de indios, negros y gauchos», Memoria \& Sociedad 9, n. 18 (2005). 
les parties du monde con seis volúmenes, en la que se pretendía abarcar la totalidad de la representación física del mundo según las condiciones de análisis creadas por los estudios y relatos de la situación política de aquel tiempo. Es lógico que al haber pretendido abarcar en una narración la complejidad de una representación total del globo hubiese sido una tarea que tenía que reinventarse, o mejor, reorganizarse continuamente para que pudiese tener un relativo éxito, pues debido a la falta de una estabilidad política en la organización de las repúblicas, reinos y territorios de diversas latitudes, era necesario que la obra se continuase reeditando en varios momentos.

La obra actualizada de mitad de siglo, en su novena edición ${ }^{18}$, contiene aspectos relevantes en la configuración de lo que se observaba dentro de la Nueva Granada. La descripción de MalteBrun presentaba a la Nueva Granada como una república de 1.800.000 individuos compuesta de blancos (hispanoamericanos y extranjeros), indios, negros u hombres de color (nègres ou hommes de couleur). Para ellos, el territorio neogranadino se dividía en tres partes: el istmo de Panamá, la región montañosa de los Andes y la planicie de los llanos al oriente. Es relevante este tipo de división pues se mantendrá en la literatura francesa frente a la Nueva Granada durante el diecinueve, de allí que en textos como El globo ilustrado de Eugene Cortambert en $1878^{19}$ se muestre como significativas para representar pictóricamente a la Nueva Granada el istmo de Panamá y los Andes $^{20}$. Se menciona que la explotación aún es negligente y no se ha aprovechado lo suficiente. Igualmente, el oro se encuentra en la cordillera central del país, en los mismos municipios. Es notable que desde allí se impartiera una visión de qué recursos son significativos para el uso de estas tierras ${ }^{21}$. Se habla de zonas que podrían superar la producción de plata del Potosí, pero no son

18 Conrad Malte-Brun, Géographie Complete Et Universelle ou description de toutes les parties du monde sur un plan nouveau.. Tome Cinquième (Paris: Eugène Et Victor Penaud Frères, Éditeurs 10, Ruedufaubourg-Montmartre, 1851-1854).

19 Eugène Cortambert, El globo ilustrado. Geografía general para uso de las escuelas y familias, arreglada para las repúblicas latinas de América, trad. César C. Guzmán (Paris: Librería Hachette y C\&., 1878). 20 Cortambert, El globo ilustrado, 48.

21 Malte-Brun, Géographie Complete, 374. explotados, y también de que las tierras altas son las más pobladas y que su mayor producción gira en torno al maíz, la yuca o cassave, el cacao, el café, el algodón, el añil, el algodón, entre otros.

En relación con la zona oriental del país, el mineral que tiene mayor expectación son las esmeraldas, de ellas se menciona lo siguiente:

El río de esmeraldas fluye desde los Andes al norte de Quito. Es en Muzo, en el valle de Tunja, cerca de Santa Fe de Bogotá, las cuales son las principales explotaciones de esmeraldas que dicen pertenecer al Perú [...]. Las que se encuentran en tumbas indígenas tienen forma de círculos, cilindros, conos y otras figuras, perforados con gran precisión; pero no se conocen los métodos que se han utilizado ${ }^{22}$.

Es interesante que las condiciones de reconocimiento de las esmeraldas se asocien con Perú, puesto que se creía que de allí salían, pero en realidad se encontraban en Muzo, en el valle de Tunja. El conocimiento de estos minerales y los componentes que los hacían particulares era notable para la época, teniendo en cuenta que el comercio de estas no había sido aún reconocido como producto de la Nueva Granada en Europa. Cabe rescatar, igualmente, que las condiciones de las esmeraldas encontradas en las tumbas de los muiscas tenían figuras que dejaban ver un trabajo esculpido, sin tener aún conocimiento de cómo o con qué técnica y herramientas se pudo haber trabajado con este mineral.

En general la visión de la provincia de Tunja y Boyacá estuvo siempre en relación con los aspectos culturales y físicos, con referencia última a la organización de la independencia y el grado de participación de esta región de la Nueva Granada en la configuración de la república, como se expresa en el siguiente texto:

Antiguamente Tunja fue rica, populosa y floreciente, cuando era una residencia del zaque o rey de la poderosa nación de los muiscas. Boyacá recuerda la victoria que, en su entorno, aseguró la causa de la independencia en 1819. Sogamoso, pequeña ciudad bastante próspera, famosa por su gran templo del Sol y el sacrificio humano de Guesa (niño pobre de quince

22 Malte-Brun, Géographie Complete, 375. (Traducción del autor). 
$\left.\operatorname{años}^{23}\right)$, que se celebra cada dos años en el momento de la dominación de los muiscas ${ }^{24}$.

Se destaca el reconocimiento de Tunja como una población populosa, floreciente y rica, allende en los tiempos de la nación muisca. Ese grado de reconocimiento es tal vez la impronta de esta región, pues depende más del símbolo que lleva consigo la disminuida cultura muisca que el presente poblacional de mediados de siglo xIx. Su gloria republicana se debe a su contexto como base de la independencia en 1819. También se menciona a Sogamoso como un lugar de implicaciones similares en importancia a Tunja, y de manera similar se considera a Pamplona y Vélez parte constitutiva de la antigua Boyacá.

Así, este tipo de visiones también se encuentran en otros documentos franceses de la época con pretensión de una narración histórica universal. Me refiero al libro Histoire de tous les peuples et des révolutions du monde depuis les temps les plus [...] jusqu'à nos jours Tome Cinquième de 1850, en el cual se vuelve a retomar estas ideas de la visión indígena como el factor principal en el reconocimiento de la provincia de Tunja y la villa de Sogamoso. No es extraño que a este tipo de narraciones pueda sumársele una especie de leyenda y fantasía en cuanto a los sacrificios rituales realizados por los muiscas, pero puede ser significativo como representación:

Tunja, ciudad que, antes de la llegada de los españoles, fue la capital de la nación muisca y que hoy no ofrece más que ruinas, mencionaremos del departamento de Boyacá la pequeña ciudad de Sogamoso, famosa por los sacrificios humanos que los muiscas celebraban allí cada quince años en honor del Sol ${ }^{25}$.

En este tipo de visión del pueblo muisca se observa cómo, a diferencia del relato anterior, se enfatiza la manera en que los sacrificios se realizaban

23 Se reconoce el sacrificio humano de Guesa como un ritual muisca que permitía crear, por medio del sacrificio de un niño de quince años criado en el templo del Sol, un nuevo ciclo de 185 lunas.

24 Malte-Brun, Géographie Complete, 388. (Traducción del autor).

25 MM. saint-Prosper, de Saurigny, Duponchel, le baron Korff, Belloc et l'abbé Martin revue et continuée par M. E. De LostalotBachoué. Histoire de tous les peuples et des révolutions du monde depuis les temps les plus [...] jusqu'à nos jours Tome Cinquième (Paris: Eugene Et Victor Penaud Frères, 1850), 341. (Traducción del autor). a los jóvenes de quince años en honor al sol, pero no se especifica el por qué y las implicaciones que esto tenía; es una representación que a primera vista reafirma la idea de lo salvaje en los muiscas y cierra las interpretaciones a la necesidad de un proceso «civilizatorio» en la región. Sin embargo, la idea de una sociedad republicana y democrática que mostraba aquel texto geográfico y universal no reproducía del todo una sociedad civilizada, sino que se mantenía en el imaginario académico francés de la percepción de lo salvaje (sauvage), de lo peligroso, no solo por las enfermedades que podrían adquirirse, sino de las condiciones de «salvajismo» en que aún se encontraban estas tierras ${ }^{26}$. Para Michel Foucault, «desde el momento en que se puede analizar el saber en términos de región, de dominio, de implantación, de desplazamiento, de transferencia, se puede comprender el proceso mediante el cual el saber funciona como un poder y reconduce a él los efectos» ${ }^{27}$. Este saber conduce, por un lado, al reconocimiento de la población, al ciframiento cada vez más detallado de la población como forma de dominio y control, el uso de la estadística como saber útil políticamente para reconocer lo que era Tunja, y, por otro lado, a la visión desde el exterior, a la identificación del territorio como forma de mantener un poder sobre los recursos que las tierras de la República de la Nueva Granada puede brindar a las potencias mercantiles y económicas de ese entonces.

En general el sentido de las representaciones geográficas desde la academia francesa sin duda alguna permite el reconocimiento de cómo se presentaba la Nueva Granada en otros entornos comunicativos. Si bien estos textos no llegaron de una forma visible a la población neogranadina, sí influyeron en la concepción y reproducción de lo que era la Nueva Granada, y en un caso específico la ville de Tunja, pues a la larga este tipo de narraciones también construyen una imagen significativa en la memoria e historia de la región.

De otro lado, una de las principales visiones norteamericanas de la Nueva Granada fue la búsqueda de apertura de rutas y uso del territorio para fines

26 Conrad Malte-Brun, Géographie universelle de Malte-Brun... Illustré par Gustave Doré (Paris: Gustave Barva, Libraire Éditeur, 1859). 27 Michel Foucault, «Preguntas a Michel Foucault sobre la geografía», en Microfísica del poder (Madrid: La Piqueta, 1979), 116. 
expansionistas de Oriente a Occidente durante la colonización que se llevó a cabo en los Estados Unidos de América antes y después de la guerra civil. En ese sentido, el aporte a la clasificación y estudio se llevó en función de consolidar su territorio mediante la navegabilidad y rastreo de recursos que pudiesen ser útiles para su economía. Como vimos en el ejemplo de la academia francesa, de manera similar los estudios sobre la Nueva Granada desde Estados Unidos estuvieron en relación con la visión política, económica y etnográfica.

A diferencia de la visión francesa en la cual el territorio de la Nueva Granada se dividía en tres partes o regiones, para Andrew Cornette en $1847^{28}$ este país se divide «naturalmente» en cinco secciones longitudinales, esto en beneficio de aquel visitante que quiera conocer este territorio. Para ellos existían ciertas precauciones antes de venir a este lugar como las siguientes:

Precauciones: Evitar la humedad del aire y del suelo, que están marcados generalmente por manantiales venenosos, y cruzar valles estrechos en la noche. Ser precavido en el uso de frutas y licores; viaje de 8 a 10 kilómetros al día. A pesar del gran cuidado y precaución, en este país la vitalidad sufrirá un gran impacto. La edad de 65 años es un máximo, y los 70 años son un fenómeno de la vida humana ${ }^{29}$.

A pesar de los problemas de humedad y la comida que pueda ingerir se recomienda como un buen lugar para la «vitalidad». En general esta entrada nos muestra una definición geográfica de la Nueva Granada con referencia a cómo alguien del norte podría considerar su estadía en el país. Se piensa que únicamente el valle del Cauca y la meseta de Bogotá son lugares habitables, los demás son espacios agrestes para la vida de un estadounidense. Lo peculiar es que se recomiendan lugares donde la comunicación puede llegar a ser mucho más difícil, y el comercio es mucho más problemático que en las zonas costeras.

De esta visión de la Nueva Granada, con las implicaciones propias de una perspectiva de estudio geográfico con fines académicos y migratorios, se analiza cuál es el interés que tiene la descripción

28 Andrew Cornette, Extract of a Geographical and physical survey made in New Granada, Central America, Mexico, Tha Antillas and United States from the year 1847 to 1868 (Spring Hill College, 1869).

29 Cornette, Extract of a Geographical, 6. (Traducción del autor). de un país como la Nueva Granada en la proyección política de Estados Unidos para el siglo xIX. Sin embargo, lo que nos compete, más que observar de forma general la visión abstracta de la Nueva Granada, es concretar aspectos relacionados con la condición del oriente del país, en especial con los intereses que este pudiera generar para Norteamérica. Uno de los documentos que puede ser significativo para entender esto es el trabajo realizado por el general Tomás Cipriano de Mosquera en 1853, Memoir on the physical and political geography of New Granada..., texto dedicado a la American Geographical and Statistical Society of New York. En este texto se puede encontrar una significativa descripción geográfica de la Nueva Granada, luego de los procesos científicos expedicionarios realizados en la comisión corográfica y trabajos adelantados durante el gobierno de dicho general en su presidencia.

Una revisión al texto puede definir la división geopolítica de la Nación. No obstante, interesa resaltar la situación de la provincia de Tunja en una sección del documento dedicado a Boyacá, y que acoge las provincias de Casanare, Tundama, Tunja y Vélez. A diferencia de las narraciones asépticas de los extranjeros, este ex presidente presenta a la Nueva Granada en un tono de revalorización de sus recursos por la exuberancia de los ríos y montañas de esta zona oriente del país. Para Mosquera las ciudades más importantes de esta región boyacense eran Tunja, Leiva, Vélez, Moniquirá, Santa Rosa, Sogamoso, Garagoa, Soata, Chiquinquirá, Guateque, Ramiriquí y el Cocuy. No obstante, ninguna de ellas tenía una organización política relevante y sus construcciones tampoco eran significativas a comparación de lugares como el Cauca, Cartagena o Bogotá. Puede rescatarse la construcción de la iglesia de Chiquinquirá y la romería que esta tiene en la región, pues implica un centro de veneración católica de gran importancia que maneja un poder de concentración importante en estas ciudades y pueblos circunvecinos. Así lo expresa el general:

[...] la más notable es la iglesia de Chiquinquirá, que es de una arquitectura sólida y muy agradable, erigida a expensas de las limosnas y donaciones de los católicos, que veneran la imagen de la Virgen pintada sobre lienzo, a la que los monjes de Santo Domingo dan extraordinario crédito, atribuyéndole milagros, la mayoría 
de los cuales son ridículos, pero esto está bien calculado para generar grandes ofrendas y regalos de los vulgares fanáticos. Para las peregrinaciones realizadas a ese santuario, y para el concurso de gente de todas partes, se debió la fundación de la villa; y, en este sentido, el santuario de Chiquinquirá ha sido útil para el país ${ }^{30}$.

Cabe resaltar que se hace un énfasis en los minerales de la región, en especial las minas de $\mathrm{Mo}^{-}$ niquirá, en Vélez, donde se trabaja apropiada y continuamente con oro, pero sobre todo resalta las minas de esmeraldas de Muzo, «the only mines of real emeralds now known in the commercial world» ${ }^{31}$. Dichas minas eran confundidas con otras formas de producción, por eso al igual que en Europa se las confundía con las de Perú, quizá por el gran valor de minerales preciosos que dicho país tuvo históricamente. Mosquera menciona algunas minas principales:

Muchos escritores sobre geografía, y otros en mineralogía, hablan de las esmeraldas del Perú, pero están en un error, ya que no están en el Perú, y nunca han estado allí; la información se deriva de la ignorancia que ha prevalecido hasta la actualidad de la geografía de América y Colombia.

Las minas de esmeraldas de Muzo, que han sido conocidas desde la conquista, tienen varias vetas a cielo abierto. Las principales son las de Camero, Perejil, Plasmera, Coronados, Juan Ignacio Camero, Hoyo Antiguo, Gerónimo Díaz, Quebrada Grande, Peñon, Quebrada Minera, Aguardiente, el Cerro, Miguel Ruiz, Agustín Camero y el Real Vena. La extensión de territorio en el que se encuentran estas minas son varias leguas; y en la provincia de Tunja, en Somondoco, otras fueron hechas en la época del gobierno colonial ${ }^{32}$.

Así, tanto en Somondoco como en Muzo la actividad minera de esmeraldas es demasiado productiva y sus productos son comercializados en el mundo pero aún sin el reconocimiento del lugar de su extracción. Pese a ello, esto no quiere decir que no exista una caracterización de las esmeraldas en cuanto a sus propiedades físicas y químicas, Mosquera retoma trabajos realizados

30 Tomás C. Mosquera, Memoir on the physical and political geography of New Granada dedicated to the American Geographical and Statistical Society of New York. By Mosquera Expresident of New Granada; Honorary Member of la Societé Agronomique Pratique de Paris; Corresponding Member of the Historical and Geographical institute of Brazil; Founding Member of the royal society of Northern Antiquaries of Denmark, \&cc, \&cc. (tr. Theodore Dwight) (New York: Publish by T. Dwight, 1853), 68. (Traducción del autor). 31 Mosquera, Memoir on the physical, 65.

32 Mosquera, Memoir on the physical, 65. (Traducción del autor). para el análisis de estas esmeraldas y presenta una clasificación química de ellas ${ }^{33}$. Este tipo de estudios consolida las visiones sobre lo relevante que era el conocimiento de la producción minera neogranadina a los ojos de los científicos europeos y norteamericanos, pero sobre todo la influencia que esto tenía en la descripción geográfica de la Nueva Granada en una visión política a mediados de siglo.

En general, el espacio percibido como una actividad imaginaria, en este caso, se realiza para contemplar el uso y apropiación de los recursos culturales y materiales de la región, que no solo implicaba a la Nueva Granada, sino que se proponía como una estrategia política universal. Esta pretensión de universalidad imprime una proyección de apropiación gubernamental que consolida formas de representación diversa al gobierno interno republicano. La percepción de las fronteras, divisiones territoriales, económicas y culturales como la construcción social en el mundo es parte de la práctica de uso, en este caso de la provincia de Tunja, adquiriendo reconocimiento económico para la academia francesa y norteamericana. No obstante, esta percepción espacial se entreteje con la misma información del gobierno provincial, adquiriendo un soporte en el diseño concebido del espacio, asegurando aspectos de transferencia para el dominio y la apropiación. Pero tanto la percepción y concepción del espacio como práctica objetiva y subjetiva se conjuga en formas «real-imaginarias» como narraciones y reminiscencias.

\section{Narración y reminiscencias como espacios vividos}

La forma de transcurrir en espacios clandestinos e imaginarios, en espacios vividos, que aparecen en la literatura académica sobre Tunja va más allá de la formación republicana y del espacio político que este quería consolidar como inicio de una nueva «civilización». Es pertinente confrontar con los estudios étnicos que daban prioridad a las huellas, a las representaciones imaginarias de la población y que consolidan lo muisca como un

33 Mosquera, Memoir on the physical, 77. 
escenario que vive y permanece a mediados del siglo XIx. Lo étnico o etnológico tiene gran significancia en la descripción de la Nueva Granada en Londres a mediados del siglo xIx. La consolidación de las disciplinas académicas y el uso de ellas en la gobernabilidad y colonización económico-política de diversas regiones del mundo son base para perfilar los desarrollos investigativos en la sociedad londinense ${ }^{34}$. Es significativo que la importancia de la expansión económica inglesa en la Nueva Granada haya empezado por la colonización de los mercados, creando dependencia mediante el consumo de mercancía inglesa, en especial sus telas ${ }^{35}$.

Un investigador londinense que logró desarrollar estudios etnológicos desde Suramérica fue William Bollaert (1801-1876), un escritor, químico, geógrafo y etnógrafo que trabajó desde muy joven, a la edad de 18 años, en Perú en la década de los treinta, y que durante los años cincuenta atraviesa los Andes desde la Nueva Granada hasta Chile, consolidando en 1860 su escrito $A n-$ tiquarian, Ethnological and other researches in New Granada, Equador, Peru and Chile, with observations on the pre-incarial, incarial, and other monuments of Peruvian nations ${ }^{36}$.

Es desde la visión de este investigador como puede observarse un tipo de clasificación de la población neogranadina. En esta descripción Bollaert toma en cuenta las caracterizaciones de los tipos

34 La importancia de la economía londinense era significativa a tal punto que las economías latinoamericanas terminaron por conducirse a su ritmo en el siglo XIX, dando inicio a la entrada del capitalismo: «Parts of Latin America, notably Argentina and Uruguay and, to a lesser extent, Brazil, were similarly placed, the rhythm of their economic life being dependent upon the ebb and flow of London funds». Anthony G. Hopkins y Peter J. Cain, «Gentlemanly Capitalism and British Expansion Overseas II: New Imperialism, 1850-1945», The Economic History Review, New Series 40, n. ${ }^{\circ}$ (1987): 11.

35 «La posición de Inglaterra como el principal proveedor de importaciones a Colombia permite preguntarse qué tipo de productos fueron importados al país. Evidentemente, lo que más se importaba de Inglaterra eran textiles de algodón y lana, que correspondían al 90 por ciento de las importaciones de este producto al país. Según los Libros de Exportaciones de Mercancías Británicas de 1873 , un total de 1'575,560 libras de algodón fueron importadas a Colombia, lo que representó más de 101'887,760 metros de tela». Ana María Otero-Cleves, «"Jeneros de gusto y sobretodos ingleses": el impacto cultural del consumo de bienes ingleses por la clase alta bogotana del siglo XIX», Historia Critica 38 (2009): 26.

36 William Bollaert, Antiquarian, Ethnological and other researches in New Granada, Equador, Peru and Chile, with observations on the pre-incarial, incarial, and other monuments of Peruvian nations (London: Trubner \& Co. Paternoster Row, 1860). raciales de seres humanos que, según él, existen en la Nueva Granada, pues no solo reafirma el número y distribución, sino que les adjudica propiedades «naturales» que identifican a la población $^{37}$. A los blancos como cuarterones y mestizos se les representa como inteligentes y activos. Es significativo encontrar que se toma una clasificación de la colonia para describir un tipo específico de blancos, que son la mayoría, pues no se le menciona como "criollo" sino como un blanco mezclado con mulato o indígena. Esto es relevante pues no se atiende a una identificación política sino a la naturalización de una especie o tipos de especie humana. En general se considera a un gran porcentaje de la población neogranadina con estas características.

Según Bollaert, los «blancos» vienen en detrimento físico y moral con las mixturas que se producen de manera continua en la Nueva Granada, lo que implica que la «naturalidad» del blanco se pierda en tres o cuatro generaciones. Así, esto conduce a que no exista un incremento en la población de blancos en esta parte del continente:

Los blancos, indios y negros al mezclarse, sobre todo en esta región, producen variedades infinitas. El señor Bollaert dijo que le parecía que esta influencia perjudicial, física y moral, ha sido producida por la mezcla de las tres especies desde alrededor de 1500, y que el cruce entre mulatos, en particular, podría generar como resultado la esterilidad. El doctor Nott observa: «Ellos mueren en tres o cuatro generaciones». Hay razones para creer que la dificultad en muchas partes del Nuevo Mundo en la crianza de los niños mestizos

37 Safford explica que la visión de lo civilizado en los geógrafos europeos tiende más a una idea económica, pues es desde el consumo como se mide el estado salvaje o civilizado: «As Colombia's midcentury geographers and other writers surveyed their country, they tended to divide its population into several categories. The first division was between the 'savage' (for the most part forest Indians) and the 'civilized,' which included sedentary Indians and Afro- Colombians as well as Europeans and mestizos. The 'civilized' in turn were divided into those who lived 'decently,' in terms of housing and clothing, and worked hard in order to obtain such 'comodidades,' and those who were satisfied with survival at a low level of material consumption and could not be induced to undertake systematic work. By and large, as elite writers saw it, the white and mestizo populations fell into the category of the meritoriously working and consuming, while Indians and Afro-Colombians in general revealed their deficient 'civilization' by being content with low levels of material consumption». Frank Safford, «Race, Integration, and Progress: Elite Attitudes and the Indian in Colombia, 17501870», The Hispanic American Historical Review, 71, n. ${ }^{1}$ (1991): 24.

Espacialidad de la provincia de Tunja en el siglo xıx: representaciones políticas y geográficas / Daniel Roberto Vega Torres / 167 
e incluso criollos es una de las causas de la falta de aumento general de la población ${ }^{38}$.

Los indios se dividen en civilizados e incivilizados, con características de ser pacientes, suspicaces y frugales (o austeros). En general aún se encontraba una cierta desconfianza a la manera como los indios o indígenas se reconocían, ya fueran civilizados o no. Era una menor parte de la población frente a los blancos. No obstante, existe una división de personas negras o de color en función de su mestizaje con indígenas o blancos. Se hace mención de que aquello que tiende a ser más africano o negro es menos «útil» que si tuviera algo de otra raza. Mientras que a los mulatos y zambos se les considera fuertes, inteligentes y osados, a los «negros etíopes» se les considera pacientes y desconfiados.

Aunque el proceso de abolición de la esclavitud en la Nueva Granada y en otros lugares del continente tuviese fuerza y consolidación a mediados del siglo XIX, es notable que si se dejaba de adquirir una sumisión a un señor o dueño como propiedad la representación de su «naturaleza» seguía siendo parte del reconocimiento poblacional del país. Es mediante estos estudios académicos que se fundamenta continuamente la valoración natural de las razas como un aspecto positivo o factual que trasciende una comprensión política y que se asegura como una estrategia de dominio de la población en función de aquello que debe ser incluido o no en las sociedades republicanas. Más que insistir en que esto es algo colonial, se reafirma que mediante el proceso de racionalización y naturalización de las especies se consolida una forma de estudio natural de la vida en América desde la clasificación racial y por ende el uso de la población en formas de producción y reproducción de conocimientos y costumbres.

No obstante, esta parte del estudio etnológico de la Nueva Granada es algo que se da por sentado, es una base donde se inicia la explicación, por ello no sería nada nuevo en la disciplina etnológica. Por el contrario, se hace énfasis en la población muisca que vivió en el centro oriente de la Nueva

38 William Bollaert, «Observations on the Past and Present Populations of the New World», Transactions of the Anthropological Society of London 1 (1863): v (5). (Traducción del autor).
Granada; es este tipo de población el que más se profundiza indagando acerca de sus costumbres, ritos, formas de organización y lengua hablada o escrita. Para el caso de Tunja, el interés sobre este territorio estaba centrado en los vestigios y antigüedades que poseían de los indígenas. Esta discusión era de relativa relevancia en la academia europea, y es desde allí donde se narra la importancia de este lugar. A continuación, se observa un texto sobre las antigüedades de la Nueva Granada, tomado del «Boletín de la Sociedad de Geografía de París»:

Las investigaciones fueron realizadas por el señor Vélez de Barrietos. Vélez fue informado de que existía en el Cantón de Leiva, en Tunja, ruinas de un templo y el palacio de los antiguos indios. Él se fue en busca de ellas en junio de 1846 . Viajó a Moniquirá (aproximadamente $6^{\circ} \mathrm{N}, 73^{\circ} 45^{\circ} \mathrm{W}$ ), y primero se encontró con columnas de piedra; una de 4 a 5 metros de largo, por $31 / 2$ de diámetro. Luego encontró 13 grandes columnas, dispuestas en un círculo de 50 metros de circunferencia, pareciéndole como si se tratará del diseño de un templo o palacio. Algunas de estas columnas eran ovaladas, como las de Ramiriquí y tenía muescas en los extremos, demostrando que habían sido arrastradas de una cantera. Cuatrocientos metros de las 13 columnas que encontraron en las ruinas principales, compuestas de columnas cilíndricas, algunas 29 en número, bien acabadas, fijas en la tierra, y que ocupan 45 metros de longitud y 22 de ancho ${ }^{39}$.

Lo anterior se ve mediante un diálogo con las sociedades de geografía en Francia, que como vimos tenía similar valor e interés etnológico. La descripción de la Nueva Granada frente a sus características etnológicas también integrará a jóvenes del notablato neogranadino como José María Samper, quién publicará en 1858 algunas consideraciones de la población neogranadi$\mathrm{na}^{40}$. En el texto de Bollaert se relacionan objetos como columnas o ruinas de templos, figuras de piedras que asemejan palacios en Moniquirá, y se reconoce un trabajo de construcción en piedra significativo para el reconocimiento de una sociedad que se pensaba no había tenido un desarrollo técnico para la construcción de palacios y una organización social demasiado desarrollada

39 Bollaert, Antiquarian, 35. (Traducción del autor).

40 José María Samper, «La Confédération Grenadine. Son territoire et sa population a la fin de 1858», Bulletín de la Societe De Geographie. Quatrième Série. Tome XVII (1858). 
como la Inca. El informe considera que puede interpretarse por la posición y forma de las columnas que efectivamente pudo presentarse una construcción desarrollada no solo en Moniquirá sino también en Ramiriquí.

Más adelante sigue su descripción de las ruinas de ciertos elementos rituales de los muiscas encontrados en la provincia de Tunja:

En el comienzo del año 1846, Vélez vio en Tunja (5 30 'S., $73^{\circ} 50^{\prime} \mathrm{W}$.) las dos piedras conocidas como «Cojines del Diablo»; ellas están en una colina seis cuadras (la cuadra son 600 metros) desde el pueblo hasta el oeste. La roca es forjada, coronada por dos piedras en forma de molino, pero un poco más grande y se asemejan a cojines. En este punto los antiguos habitantes pueden haber adorado al sol naciente.

Nuestro explorador luego pasó por Boyacá a $\mathrm{Ra}^{-}$ miriquí para examinar las grandes columnas (Boyacá está a pocos kilómetros de Santiago de Tunja) conocidas como «Vigas del Diablo». A poca distancia del río de Ramiriquí, donde encontraron grandes columnas elípticas puestas en la tierra, de unos 7 $1 / 4$ metros de longitud; en sus extremidades tenían muescas para ayudar en el transporte en la cantera. Otra columna era de $4 \frac{1}{2} 2$ metros de largo, no cilíndrico, pero con varios lados. El cura de Ramiriquí le comentó a Vélez que en una parte de la parroquia había cinco o seis columnas similares ${ }^{41}$.

Las piedras como «Cojines del Diablo» en Tunja o las «Vigas del Diablo» en el pueblo de Boyacá pueden llegar a ser de gran expectación para Bollaert, pues podrían representar objetos de adoración de los antiguos indígenas ${ }^{42}$, que aunque no tenían todas estas rocas unas figuras definidas, sí daba a la interpretación que habían sido construidas con fines específicos. En general, este tipo de indagaciones sobre las condiciones de la provincia de Tunja permiten la construcción de una visión de allí desde otros lugares o focos de observación,

41 Samper, «La Confédération Grenadine», 36. (Traducción del autor). 42 Estas ruinas o construcciones en piedra no podrían denominarse descubrimientos de extranjeros, pues tanto los pobladores de estas tierras, cronistas en la colonia, al igual que militares y políticos neogranadinos ya habían relatado la existencia de esta especie de «templos». Es el caso de Alejandro Vélez Barrientos: «Recordamos aquí las ruinas de un antiguo templo, cuyas grandes columnas él encontró en la provincia de Tunja, muchas en sus puestos, pero otras que iban rodando en pedazos y fuera de su lugar, cediendo ya al impulso de los años y a la descomposición de la materia como también a la mano destructora del hombre ignorante. Este descubrimiento no ha muchos años se hizo público y ha sido el primer paso, a nuestro saber, que se ha dado sobre esta materia y que siempre recordaremos con placer». Ezequiel Uricoechea, Antigüedades neogranadinas (Bogotá: Ed. Minerva, 1870): 28-29. tanto reales como imaginarios, pues se alejan de las representaciones político-administrativas, y adquieren un carácter de viaje por lugares y escenarios en ruinas, mistificados por la narración popular y la medición académica. La especialización en la ubicación y el reconocimiento cultural de los habitantes consigue integrar las mixturas de tiempos que se entrecruzan, pues las «Piedras del Diablo» pueden ser concebidas como puntos de referencia o signos de la región para la localización de la cultura muisca que no contempla la cartografía política del momento.

También el geógrafo alemán Alfred Hettner en su temporada en Colombia de 1882 a 1884, mencionaba la existencia de estos restos chibchas:

Ya los chibchas tenían aquí la ciudad como residencia de su poderoso zaque. Todavía hoy día se encuentran encima de las colinas al oeste de Tunja, cerca del camino que conduce a Leiva, colocadas sobre un banco de arenisca ferruginosa inclinado hacia el este, dos lajas de arenisca en forma circular a la manera de molares, pero con faceta oblicua en el borde superior, los llamados «cojines», sobre los cuales los indios antiguos dizque arrodillados solían elevar sus plegarias con la cara vuelta hacia el oriente. Se dice que cerca de aquellas piedras hace poco todavía estaban a la vista las ruinas del palacio del zaque ${ }^{43}$.

Por un lado, la visión de este extranjero alemán puede describir los espacios de ubicación culturalmente inscritos en las huellas escultóricas. No obstante, la visión de él acerca de la ciudad de Tunja es la de un lugar inigualable en el país por su número de iglesias «con un aburrido estilo tradicional», las cuales a falta de fondos se deterioraban, con pocas casas de aspecto sólido y acogedor, y en general se presentaba el desaseo en las calles, lo que generaba plagas y un espacio de vida deplorable. Puede sobreponerse en un espacio los tiempos que constituyen las representaciones vivas de la cultura muisca en la región.

Un espacio vivido, constituido por diversas narraciones que se contraponen a la literatura cronológica y lineal, puede generar una reflexión alterna, que se guía más por la memoria y las proyecciones que por los hechos son contra-espacios que

43 Alfred Hettner, Viajes por los Andes colombianos: (1882-1884) (Bogotá: Banco de la República, 1976). Biblioteca Virtual BLAA. http://www.banrepcultural.org/blaavirtual/historia/viaand/indice. htm (consultado el 20 de septiembre de 2014). 
se alternan sobre símbolos, indicaciones, huellas que conviven con el presente. Una búsqueda por la localización popular, un viaje por el territorio, una experiencia que permite hablar de lo que no es explícito o «normal» sino que es una propuesta «real-imaginaria» de ubicación.

\section{A manera de conclusión: representaciones de Tunja}

Uno de los inconvenientes que presenta la reproducción abstracta de la representación concebida o diseñada, sea por organismos gubernamentales o alternos, es el de seguir tomando de forma plana la descripción del espacio como una condición analítica más que como una condición histórica. Así, en este texto se ha intentado mostrar el espacio social de la provincia de Tunja desde una reflexión diferente, sustentada por representaciones reales e imaginadas, por diversas visiones que constituyen tanto realidades espaciales que se entrecruzan en tiempos, como espacios que definen las duraciones. De esta forma, se inicia la reflexión por las implicaciones político-administrativas de dominio de un territorio en un escenario de conflicto social reiterado, del que se generan diseños que se consolidaban en las cartografías de los gobiernos republicanos, que si bien corresponde como parte de la literatura geográfica, es necesario observarla como una práctica social que representa el espacio, más que el espacio físico mismo.

Esta diferenciación propende por entender que el espacio puede ser investigado por percepciones e imaginarios que definen otro tipo de espacios, ya no como diseño sino como práctica espacial, formas de ver subjetivamente la realidad y que tiene un uso diferente, complementando de manera analítica la objetividad que proyecta el espacio concebido. Así, el uso de la geografía universal para el reconocimiento de la provincia de Tunja, tanto en Francia como en Norteamérica, contribuye a crear formas de apropiación del territorio con intenciones claras de dominio y emplazamiento. La percepción académica y política de los recursos económicos y culturales puede llegar a obtener una representación concreta de lo que era Tunja según indicadores definidos para obtener una apropiación eficiente.
Por último, existen espacios que se transitan fuera de las convenciones prefiguradas del dominio gubernamental republicano y de las percepciones académicas y geográficas. Son contra-espacios que se transponen en la realidad, que conviven en las reminiscencias y memorias de los habitantes, pues no vivimos en un espacio «neutro y blanco», ni en «el rectángulo de una hoja de papel» ${ }^{44}$. Un espacio vivido se constituye de una práctica social heterotópica, en lugares que persisten en el tiempo gracias al recuerdo, a la localización cultural e identificación social. Este es el caso de la provincia de Tunja como lugar de memoria muisca, de convivencia con los límites, símbolos de organización espacial y cultural que convive con la gran cantidad de iglesias y centros republicanos, un lugar que atraviesa lo visual y se inscribe en las visiones y memorias, generando espacios de representación que se inscriben como «real-imaginarios».

\section{Bibliografía}

\section{Fuentes primarias}

Archivo General de la Nación (AGN). Carta corográfica de la provincia de Tunja, J. F. M. a expensas de Francisco José de Hoyos, lo hizo marzo 15 de 1843. En colores 89 X $63 \mathrm{~cm}$. Escala de 10 leguas. Mapoteca 6, ref. 91. Núm. 1814.

Archivo General de la Nación (agN). Provincia de Tunja. Mapa corográfico de la provincia de Tunja, levantado por orden del gobierno por Agustín Codazzi, 1850. 64x68. Escala: 1: 340.000. Mapoteca: smp 6, Ref. 20.

Archivo General de la Nación (AgN). Tierras vecinas a la parroquia de Toca (1806). Mapoteca, SMP. 4, Ref. 482 A.

Bollaert, William. «Observations on the Past and Present Populations of the New World». Transactions of the Anthropological Society of London 1 (1863): 72-119.

Bollaert, William. Antiquarian, Ethnological and other researches in New Granada, Equador, Peru and Chile, with observations on the pre-incarial,

\footnotetext{
44 Michel Foucault, «Topologías», Revista Fractal 48 (1966/2008): 39. http://www.mxfractal.org/RevistaFractal48MichelFoucault.html (consultado en octubre de 2014).
} 
incarial, and other monuments of Peruvian nations. London: Trubner \& Co. Paternoster Row, 1860. Cornette, Andrew. Extract of a Geographical and physical survey made in New Granada, Central America, Mexico, Tha Antillas and United States from the year 1847 to 1868. Alabama: Spring Hill College, 1869.

Cortambert, Eugène. El globo ilustrado. Geografía general para uso de las escuelas y familias, arreglada para las repuiblicas latinas de América, traducido por César C. Guzmán. Paris: Librería Hachette $\mathrm{y}$ C\&. 1878.

Estadística Jeneral de la Nueva Granada. Parte Primera. Bogotá: Biblioteca Nacional de Colombia (вNC), Fondo Pineda 486. 1846.

Malte-Brun, Conrad. Géographie Complete Et Universelle ou description de toutes les parties du monde sur un plan nouveau... Tome Cinquième. Paris: Eugène Et Victor Penaud Frères, Éditeurs 10, Ruedufaubourg-Montmartre, 1851-1854.

Malte-Brun, Conrad. Géographie universelle de Malte-Brun... Illustrépar Gustave Doré. Paris: Gustave Barva, Libraire Éditeur, 1859.

Memoria del gobernador de Tunja a la cámara provincial. Bogotá: Biblioteca Nacional de Colombia, Fondo Pineda 733, pza. 25, 1843.

MM. saint-Prosper, de Saurigny, Duponchel, le baron Korff, Belloc et l'abbé Martin revue et continuée par M. E. De Lostalot-Bachoué. Histoire de tous les peuples et des révolutions du monde depuis les temps les plus [...] jusqu'à nos jours Tome Cinquième. Paris : Eugene Et Victor Penaud Frères, 1850.

Moreno y Escandón, Francisco Antonio. Indios y Mestizos de la Nueva Granada a finales del siglo XVIII. Transcripción de Germán Colmenares y Alonso Valencia. Bogotá: Biblioteca Banco popular, 1985.

Mosquera, Tomás C. Memoir on the physical and political geography of New Granada dedicated to the American Geographical and Statistical Society of New York. By Mosquera Expresident of New Granada; Honorary Member of la Societé Agronomique Pratique de Paris; Corresponding Member of the Historical and Geographical institute of Brazil; Founding Member of the royal society of Northern Antiquaries of Denmark, $\xi_{c}$, $\xi_{c}$. (tr. Theodore Dwight). New York: Publish by T. Dwight, 1853. Samper, José María. «La Confédération Grenadine. Son territoire et sa population a la fin de 1858». En Bulletín de la Societe De Geographie. Quatrième Série. Tome хиII (1858): 207-211.
Soja, Edward W. Thirdspace: Journeys to Los Angeles and other Real-and-Imagined Places. Oxford: Blackwell, 1996.

\section{Fuentes secundarias}

Bolívar Ramírez, Ingrid. «Los viajeros del siglo XIX y el "proceso de la civilización": Imágenes de indios, negros y gauchos». Memoria E Sociedad 9, n. ${ }^{\circ} 18$ (2005): 19-32.

Cortés de Sarmiento, Clara. Dimensión espacial de Boyacá: geografía. Tunja: Academia Boyacense de Historia, 2008.

Duque Muñoz, Lucía. «Geografía y cartografía en la Nueva Granada (1840-1865): Producción, clasificación temática e intereses». Anuario Colombiano de Historia Social y de la Cultura 33 (2006): 11-30.

Duque Muñoz, Lucía. «El discurso geográfico y cartográfico colombiano sobre los límites entre Nueva Granada y Venezuela (1830-1883)». Anuario Colombiano de Historia Social y de la Cultura 36, n. 1 (2009): 125-152.

Foucault, Michel. «Preguntas a Michel Foucault sobre la geografía». En Microfísica del poder, 111124. Madrid: La Piqueta, 1979, 111-124.

Foucault, Michel. «Topologías», Revista Fractal 48 (1966/2008): 39. http://www.mxfractal.org/RevistaFractal48MichelFoucault.html. Consultado: en octubre de 2014.

Herrera Ángel, Marta. «Las divisiones político-administrativas del virreinato de la Nueva Granada a finales del período colonial». Historia Crítica, n. 22 (2001): 76-98.

Hettner, Alfred. Viajes por los Andes colombianos: (1882-1884). Bogotá: Banco de la República, 1976. Biblioteca Virtual вlAa. http://www. banrepcultural.org/blaavirtual/historia/viaand/ indice.htm. Consultado: 20 septiembre de 201.

Hopkins, Anthony G. y Peter. J. Cain. «Gentlemanly Capitalism and British Expansion Overseas II: New Imperialism, 1850-1945». The Economic History Review, New Series 40, n. 1 (1987): 1-26. Instituto Geográfico Agustín Codazzi. Características geográficas Boyacá. Bogotá: Instituto Geográfico Agustín Codazzi (IGAC), 1990.

Jaramillo Uribe, Jaime. «La visión de los otros. Colombia vista por observadores extranjeros en el siglo XIX». Historia crítica 24 (2003): 7-26.

Espacialidad de la provincia de Tunja en el siglo xix: representaciones políticas y geográficas / Daniel Roberto Vega Torres / 171 
Martínez, Martha y Luz Marina Bautista. Cartografía histórica de los territorios boyacenses. Tunja: Banco de la Republica, 2003.

Otero-Cleves, Ana María. «"Jeneros de gusto y sobretodos ingleses": el impacto cultural del consumo de bienes ingleses por la clase alta bogotana del siglo XIX». Historia Critica 38 (2009): 20-45.

Patiño, Víctor Manuel. La tierra en la América Equinoccial, Libro Iv. Bogotá: Presidencia de la República, 1997.

Rincón, Carlos y Volker Lühr. «Del poder y la geografía: La cartografía como fuente de legitimación en Colombia». Disertación inaugural para la obtención del título de doctor en la Facultad de Filosofía y Humanidades, Universidad Libre de Berlín, 2006.

Safford, Frank. «Race, Integration, and Progress: Elite Attitudes and the Indian in Colombia,
1750-1870». The Hispanic American Historical Review 71, n. 1 (1991): 1-33.

Uricoechea, Ezequiel. Antigüedades neogranadinas. Bogotá: Ed. Minerva, 1870.

Fecha de recepción: 30 de septiembre de 2014

Fecha de aprobación: 7 de abril de 2015

Disponible en línea: 30 de agosto de 2015

\section{Cómo citar este artículo}

Vega Torres, Daniel Roberto. «Espacialidad de la provincia de Tunja en el siglo xIx: representaciones políticas y geográficas». Memoria y Sociedad 19, n. $^{\circ}$ 39 (2015): 157-172. http://dx.doi.org/10.11144/ Javeriana.mys19-39.epts 